\title{
MicroRNA-125 inhibits RKO colorectal cancer cell growth by targeting VEGF
}

\author{
QI-BING WU ${ }^{1 *}$, JING CHEN $^{2,3^{*}}$, JIA-WEI ZHU ${ }^{2 *}$, XIN YIN $^{2}$, \\ HE-YANG YOU ${ }^{2}$, YI-RONG LIN ${ }^{2}$ and HUA-QING ZHU ${ }^{2}$
}

\author{
${ }^{1}$ Department of Radiotherapy, The First Affiliated Hospital of Anhui Medical University, Hefei, Anhui 230022; \\ ${ }^{2}$ Laboratory of Molecular Biology and Department of Biochemistry, Anhui Medical University, Hefei, Anhui 230032; \\ ${ }^{3}$ Department of Urology, The First Affiliated Hospital of Anhui Medical University, Hefei, Anhui 230022, P.R. China
}

Received June 22, 2017; Accepted April 3, 2018

DOI: $10.3892 /$ ijmm.2018.3632

\begin{abstract}
Colorectal cancer (CRC) is one of the major types of cancer and causes of mortality worldwide, and it remains the third most common cause of cancer-associated mortality worldwide. MicroRNAs (miRNAs) are a class of small RNAs, which have been shown to be associated with CRC. In the present study, an MTT assay and proliferating cell nuclear antigen (PCNA) protein examination assay were performed to detect RKO cell viability. Hoechst staining, and caspase-3 activity and BrdU incorporation assays were performed to detect RKO cell apoptosis, respectively. Reverse transcription-quantitative polymerase chain reaction (RT-qPCR) and western blot analyses were used to analyze the expression of cyclooxygenase-2 (COX-2). Western blot analysis was also used to analyze the expression of vascular endothelial growth factor (VEGF) and mitogen-activated protein kinase (MAPK) signal molecules, including extracellular signal-regulated kinase (ERK), p38 and c-Jun N-terminal kinase (JNK). The target genes of miR-125 were predicted using a double luciferase reporter gene assay. The results of the MTT assay showed that RKO cell viability was decreased by an miRNA125 mimic and increased by the miRNA-125 inhibitor. The RKO cell viability was significantly correlated with the expression of PCNA. The migration of RKO cells was significantly downregulated in the miR-125 mimics-transfected cells and upregulated in the miRNA-125 inhibitor-transfected cells. The results of Hoechst staining and the caspase-3 activity and BrdU incorporation assays showed that RKO cell apoptosis was increased following miRNA-125 mimic transfection and
\end{abstract}

Correspondence to: Professor Hua-Qing Zhu, Laboratory of Molecular Biology and Department of Biochemistry, Anhui Medical University, 81 Meishan Road, Hefei, Anhui 230032, P.R. China

E-mail: aydzhq@126.com

*Contributed equally

Key words: microRNA-125, colorectal cancer, vascular endothelial growth factor decreased following miRNA-125 inhibitor transfection. The results of the RT-qPCR and western blot analysis showed that the expression of COX-2 was increased in the miR-125 mimic-transfected cells and decreased in the miR-125 inhibitor-transfected cells. Using an online miRNA target prediction database, the double luciferase reporter gene assay showed that miR-125 targeted and inhibited the expression of VEGF through target sites located in the $3^{\prime}$ untranslated region of VEGF mRNA. In conclusion, the abnormal expression of miR-125 was found to be closely associated with CRC. Therefore, miR-125 may be a novel therapeutic target for CRC.

\section{Introduction}

Colorectal cancer (CRC) is one of the major types of cancer and causes of mortality worldwide, and it remains the third most common cause of cancer-associated mortality worldwide $(1,2)$. Oncogenes and tumor suppressor genes, accompanied by deregulated gene expression owing to epigenetic changes may be involved in the mutational events in the development and progression of CRC (3).

MicroRNAs (miRNAs) are a class of small RNAs, $\sim 22$ nucleotides long, which are non-coding and control gene expression through the repression of target messenger RNAs (mRNAs) (4,5). The miRNAs negatively regulate gene expression by the degradation or translational repression of a target mRNA by targeting the 3 ' untranslational region (3'UTR) of specific mRNAs (6,7). miRNAs are associated with the majority of pathophysiological cellular processes, including development, differentiation, growth, migration and apoptosis (8-10). miR-125, a highly conserved miRNA, is transcribed from three different clusters and has been shown to be dysregulated in multiple malignancies through the suppression of multiple targets (11). Martínez-Acuña et al determined the expression of miR-125a-5p from nine cervical cell lines by reverse transcription-polymerase chain reaction (RT-PCR) analysis and found that miR-125a-5p was involved in the migration of tumor cervical cancer cells by acting on mitogen-activated protein kinase (MAPK)1 as a functional target (12). miR-125 is also involved in cancer inflammation by the regulating the expression of cyclooxygenase-2 (COX-2), which is an important target in various types of tumor (13). 
It is known that the occurrence of CRC is associated with multiple signaling pathways. miRNAs have shown promise in the development of drugs targeting the specific novel pathways associated with cancer. For example, anti-angiogenic therapies are being used more frequently in the treatment of CRC. Vascular endothelial growth factor (VEGF) is a number of the platelet-derived growth factor family. It includes related glycoproteins, including VEGF-A, VEGF-B, VEGF-C and VEGF-D, which are coded by genes located on 6p23.1, 11q3.3, $4 q 34.3$ and Xp22.31, respectively, with differing functions and receptors (12). A previous study found that miR-126 offers therapeutic potential in CRC through targeting VEGF-A and the subsequent regulation of angiogenesis (14). From results of a meta-analysis, it has been found that the high expression of miR-125b may predict poor survival rates in CRC, non-small cell lung cancer and prostate cancer (15). Bi et al (16) found that miR-125a suppressed tumor cell proliferation by inhibiting the expression of VEGF-A. The low expression of miR-125a in hepatocellular cancer was offset by transfected cells with small interfering RNAs inhibiting the expression of VEGF-A. However, whether miR-125 affects CRC through the targeting of VEGF remains to be elucidated. In the present study, a dual-luciferase reporter assay was used to detect the level of miR-125 in order to assess whether miR-125 affects CRC through the targeting of VEGF. The aim of the present study was to investigate the role of miRNA-125 in CRC. Therefore, the effect of miR-125 on the development of CRC was investigated by examining the expression of VEGF, COX-2 and the MAPK signaling pathway.

\section{Materials and methods}

Materials. IMDM was obtained from Gibco; Thermo Fisher Scientific, Inc. (Waltham, MA, USA). Fetal bovine serum was purchased from Zhejiang Tianhang Biological Technology, Co., Ltd. (Zhejiang, China). miR-125 mimic and inhibitor were obtained from Qiagen China Co., Ltd. (Shanghai, China).

Cell line and cell culture. The RKO cell line was obtained from American Type Culture Collection (Manassas, VA, USA) and was cultured at $37^{\circ} \mathrm{C}$ in IMDM $(4,500 \mathrm{mg} / 1 \mathrm{D}$-glucose, $110 \mathrm{mg} / 1$ sodium pyruvate, $25 \mathrm{mM}$ HEPES (5.958 g/l), $584 \mathrm{mg} / \mathrm{l} \mathrm{L}$-glutamine, $3.024 \mathrm{~g} / 1 \mathrm{NaHCO}_{3}, 15 \mathrm{mg} / \mathrm{l}$-phenol red), which was supplemented with $10 \%$ fetal bovine serum, $100 \mathrm{U} / \mathrm{ml}$ penicillin and $100 \mathrm{U} / \mathrm{ml}$ streptomycin in a $5 \% \mathrm{CO}_{2}$ incubator. The medium was replaced every 2 or 3 days.

Modulation of miR-125. An miR-125 mimic and antagomir were used to overexpress or inhibit the expression of miR-125, respectively. A scrambled oligonucleotide (GenePharm, Inc., Sunnyvale, CA, USA) was used as a control. Transfection was performed using TransMessenger transfection reagent (Qiagen GmbH, Hilden, Germany) following the manufacturer's protocol.

miR-125 expression assay. Total RNA form the RKO cells was extracted using TRIzol reagent (Invitrogen; Thermo Fisher Scientific, Inc.). The expression of miR-125 was determined using an miRNA plate assay kit (Signosis, Inc., Santa Clara, CA, USA) in accordance with the manufacturer's protocol. The
U6 snRNA was selected as an internal control to normalized RNA content.

MTT assay. The cell cytotoxicity was evaluated using an MTT assay. Briefly, the RKO cells, which had been transfected with the miR-125 mimic or inhibitor or control miR-125, were seeded into 96-well culture plates (Costar; Corning Incorporated, Corning, NY, USA) at a density of $1 \times 10^{6}$ cells/well and incubated with $5 \% \mathrm{CO}_{2}$ at $37^{\circ} \mathrm{C}$. The MTT solution (Sigma-Aldrich; EMD Millipore, Billerica, MA, USA) was added into the 96 -well plates (final concentration of $5 \mathrm{mg} / \mathrm{ml}$ ), and incubated at $37^{\circ} \mathrm{C}$ for $4 \mathrm{~h}$. Following incubation, $150 \mu 1$ DMSO (Amresco, Inc., Solon, OH, USA) was added into the 96-well plates to dissolve the crystal and incubated for $15 \mathrm{~min}$. Finally, the optical density values of the obtained solution were examined using an enzyme-linked immunosorbent assay (ELISA) reader (Thermo Fisher Scientific, Inc.) at the wavelength of $490 \mathrm{~nm}$.

Flow cytometry. The cells were fixed with $4 \%$ paraformaldehyde and were permeabilized using $0.1 \%$ Triton X-100. Following washing with PBS three times. The apoptosis assay was performed according to the manufacturer's protocol. Briefly, the cells were washed and suspended in binding buffer and incubated with $10 \mu \mathrm{l}$ of Annexin $\mathrm{V}$ for $10 \mathrm{~min}$ and $10 \mu \mathrm{l}$ of propidium iodide for $5 \mathrm{~min}$. Following two washes in PBS, the cells were suspended in binding buffer and analyzed immediately using the FACSCanto II flow cytometer (BD Biosciences, Franklin Lakes, NJ, USA) and analyzed using FlowJo software version 7.6.1 (Tree Star, Inc., Ashland, OR, USA).

Caspase-3 activity measurement. RKO cell caspase-3 activity was measured using a caspase-3 fluorescent assay kit (Enzo Life Sciences, Inc., Farmingdale, NY, USA).

Measurement of cellular DNA fragmentation. The RKO cells were seeded at a density of $1 \times 10^{6}$ cells/well and incubated overnight at $37^{\circ} \mathrm{C}$. Then, the RKO cells were pre-labeled with $\mathrm{BrdU}(15 \mathrm{ng} /$ well) and then incubated with palmitate (500 $\mu \mathrm{l} /$ well) for $4 \mathrm{~h}$ at room temperature. DNA fragmentation was measured in accordance with the manufacturer's protocol of the Cellular DNA Fragmentation ELISA kit (Roche Applied Science, Penzberg, Germany).

Western blot analysis. The protein levels of VEGF, COX-2, PCNA and GAPDH were determined using western blot analysis. The protein samples were extracted from the cultured RKO cells which were treated with a lysis buffer containing protease and phosphatase inhibitors (1 M Tris- $\mathrm{HCl}, 5 \mathrm{M}$ $\mathrm{NaCl}, 10 \% \mathrm{NP}-40,10 \% \mathrm{Na}$-deoxycholate, 100 mM EDTA, $0.1 \%$ aprotinin, $0.1 \%$ leupeptin, $0.1 \%$ pepstatin, $0.5 \%$ PMSF, $0.5 \% \mathrm{Na}_{3} \mathrm{Vo}_{4}$ and $0.5 \% \mathrm{NaF}$ ) and the protein concentrations were determined using a BCA protein assay. The quantity of protein loaded for separation was $1 \mathrm{mg} / \mathrm{ml}$. Protein $(20 \mu \mathrm{l})$ were separated by $12 \%$ SDS-PAGE gel and electrophoretically transferred onto polyvinylidenefluoride membranes. Following blocking with 5\% non-fat dry milk for $2 \mathrm{~h}$ at a room temperature, the membranes were washed with TBS-Tween-20 [150 mM Nacl, $50 \mathrm{mM}$ Tris (pH 7.5) and 0.1\% Tween-20] and incubated with primary antibodies against VEGF (1:1,000; cat. 
no. 2463), COX-2 (1:1,000; cat. no. 4842), PCNA (1:1,000; cat. no. 13110) and GAPDH (1:1,000; cat. no. 5174) (all from Cell Signaling Technology, Inc., Danvers, MA, USA) overnight at $4^{\circ} \mathrm{C}$, and then incubated with the horseradish peroxidase-conjugated secondary antibodies (1:5,000; cat.no. ZB-2301; go at anti-rabbit; Bejing Zhongshan Jinqiao Biotechnology Co., Ltd., Beijing, China) for $1 \mathrm{~h}$ at room temperature. Proteinbands were visualized by enchanced chemiluminescence using ECL reagents (Pierce; Thermo Fisher Scientific, Inc.). GAPDH was used as the internal reference. Blots were semi-quantified by densitometric analysis using the Image-Pro Plus software version 6.0 (Media Cybernetics, Ine., Rockville, MD, USA).

Reverse transcription-quantitative polymerase chain reaction $(R T-q P C R)$ analysis. Using TRIzol reagent (Invitrogen; Thermo Fisher Scientific, Inc.), the RNA was extracted from the RKO cells and was reverse transcribed into complementary DNA using the TransScript first-strand cDNA synthesis kit in accordance with the manufacturer's protocol (Qiagen $\mathrm{GmbH}$ ). The following primers were used: COX-2, forward, 5'-TTA CAATGCTGACTATGGCTAC-3' and reverse, 5'-CTGATG CGTGAAGTGCTG-3'; GAPDH, forward, 5'-GGAGCGAGA TCCCTCCAAAAT-3' and reverse, 5'-GGCTGTTGTCAT ACTTCTCATGG-3'. The total volume of PCR reaction was $20 \mu \mathrm{l}: 10 \mu \mathrm{l}$ DNA Master SYBR green I (Finnzymes OY, Espoo, Finland), $0.6 \mu \mathrm{l}$ forward primer and $0.6 \mu \mathrm{l}$ reverse primer, $4 \mu \mathrm{l}$ cDNA, $4.8 \mu \mathrm{l}$ RNase-free water. The following thermocycling conditions were used for the PCR: Initial denaturation at $95^{\circ} \mathrm{C}$ for $15 \mathrm{~min}$; 40 cycles of denaturation at $95^{\circ} \mathrm{C}$ for $10 \mathrm{sec}$, annealing at $60^{\circ} \mathrm{C}$ for $30 \mathrm{sec}$ extension at $72^{\circ} \mathrm{C}$ for $30 \mathrm{sec}$. Using GraphPad Prism software version 5.01 (GraphPad Software, Inc., La Jolla, CA, USA), relative gene expression was quantified according to the comparative $\mathrm{Cq}$ method (17). With respect to the expression levels of GAPDH, all results were normalized.

Luciferase assays. The wild-type luciferase vector (wt-LucVEGF) contained has-miR-125 response elements in the 3'UTR of VEGF. Putative binding sites located in the 3'UTR of VEGF were identified using TargetScan version5 (www. targetscan.org/vert_5). The mutant (mu-Luc-VEGF) vector with a mutation in the has-miR-125 response elements was generated using site-directed gene mutagenesis. The reporter vector, which consisted of a luciferase gene followed by the miR-125 binding consensus sequence, was obtained from Signosis, Inc. (Sunnyvale, CA, USA). The RKO cells were cultured for $24 \mathrm{~h}$. To treat cells with a miR-125 mimic or a control oligonucleotide, the cells were transfected with $200 \mathrm{ng}$ of plasmid DNA (wt-Luc-VEGF or mu-Luc-VEGF) using Attractene transfection reagent (Qiagen $\mathrm{GmbH}$ ) in accordance with the manufacturer's protocol. The $\mathrm{pRL}-\mathrm{CMV}$ vector served as an internal control by expressing Renilla luciferase. At $24 \mathrm{~h}$ post-transfection, luciferase assays were performed using the dual luciferase reporter assay system (Promega Corporation, Madison, WI, USA).

Statistical analysis. The data are expressed as the mean \pm standard deviation. Two-way analysis of variance was used to assess the statistical significance of the differences between groups followed by Student-Neuman-Keuls test for

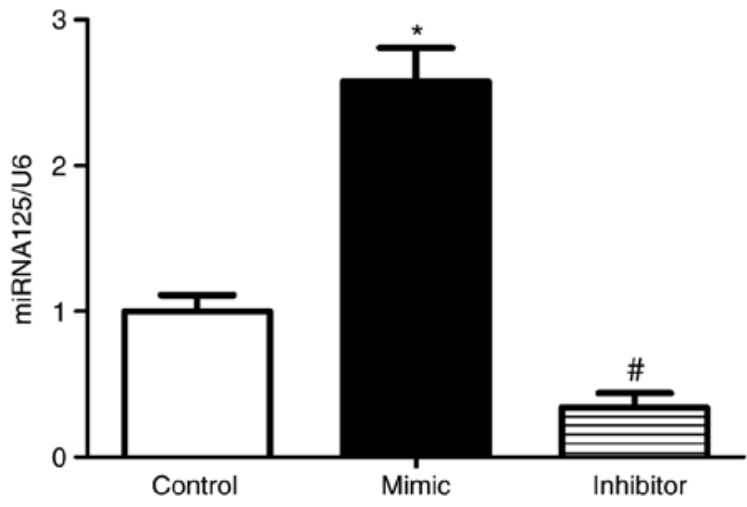

Figure 1. Expression of miR-125 in RKO cells. RKO cells were transfected with miR-125 mimic, inhibitor, or miR-125 negative control. The miRNA plate assay showed that the miR-125 mimics and miR-125 inhibitors had been successfully transfected. ${ }^{*} \mathrm{P}<0.05$ vs. control; ${ }^{\#} \mathrm{P}<0.05$ vs. control. $\mathrm{miR} / \mathrm{miRNA}$, microRNA.

multiple comparisons. $\mathrm{P}<0.05$ was considered to indicate a statistically significant difference. Statistical analysis was performed using SPSS software version 21.0 (IBM Corporation, Armonk, NY, USA).

\section{Results}

To assess the effect of miR-125 in RKO cells, the RKOs cells were transfected with miR-125 mimic or inhibitor or control miR. After 24 h, miR-125 was determined in the RKO cells using the miRNA plate assay. The level of miR-125 was increased in the RKO cells transfected with miR-125 mimic, compared with that in the RKO cells. By contrast, the expression of miR-125 was significantly downregulated in RKO cells transfected with miR-125 inhibitor (Fig. 1).

To determine the effect of miR-125 on the viability of RKO cells, the RKO cells were transfected with miR-125 mimic or inhibitor or control miR. After $0,12,24,36,48,60$ and $72 \mathrm{~h}$, the number and absorptivity of the cells were determined using MTT and DMSO, respectively. The absorptivity of RKO cells transfected with mimics was decreased and the absorptivity of RKO cells transfected with inhibitor was increased, compared with the control (Fig. 2A). Following transfection of the RKO cells with miR-125 mimics or inhibitors or control miR, the expression of PCNA was determined by western blot analysis. The protein level of PCNA was decreased in the RKO cells transfected with mimics, compared with that in the control cells (Fig. 2B). Compared with the control, whereas the protein levels of PCNA were increased in the RKO cells transfected with inhibitors (Fig. 2C). This showed that miR-125 significantly altered the expression of PCNA in the RKO cells. The growth of the RKO cells was also measured by flow cytometry. The results showed that the population of RKO cells transfected with mimics was decreased and the population of RKO cells transfected with inhibitor was increased, compared with the population in the control (Fig. 2D).

To investigate the role of miR-125 in RKO cell migration, following transfection of the RKO cells with miR-125 mimics or inhibitors or control $\mathrm{miR}$, cell scratches were observed at 0 and $48 \mathrm{~h}$, respectively. Compared with the negative control, only a few cells migrated into the scratch wound in the 
A

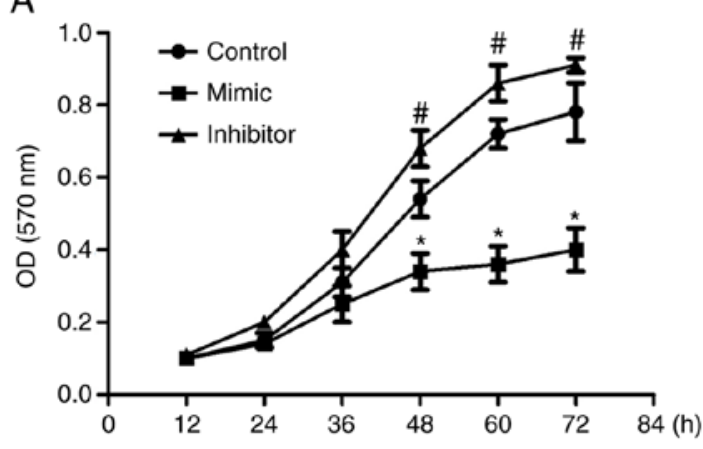

B
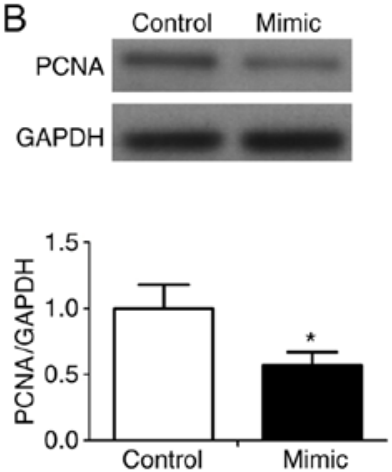
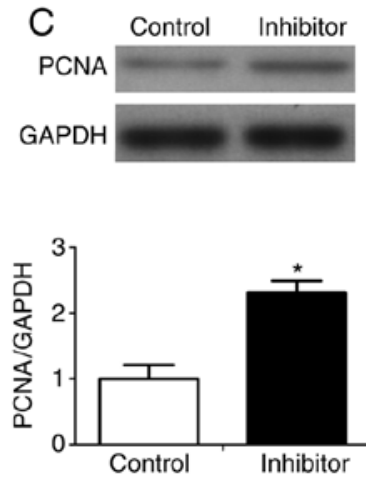

Inhibitor
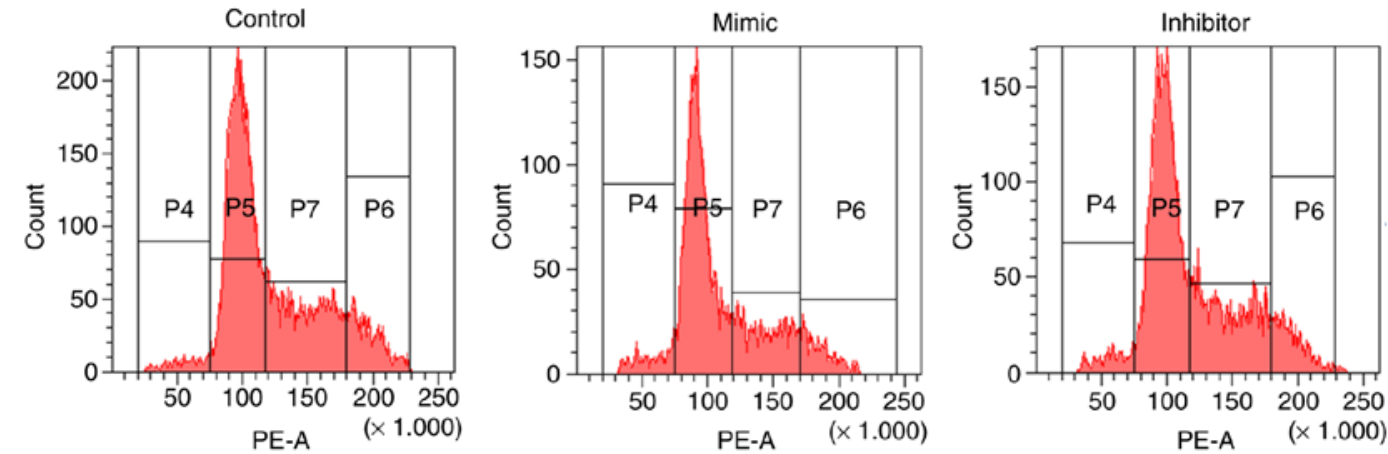

Figure 2. Effect of miR-125 on the cytotoxicity of RKO cells. RKO cells were transfected with miR-125 mimic, inhibitor, or miR negative control. (A) After $0,12,24,36,48,60$ and $72 \mathrm{~h}$ the number and absorptivity of cells were determined using MTT and DMSO, respectively. (B) Western blot analysis was performed to detect the expression and (C) levels of PCNA and GAPDH. (D) Growth of RKO cells was measured by flow cytometry. "P<0.05 vs. control; ${ }^{\#} \mathrm{P}<0.05$ vs. control. miR, microRNA; PCNA, proliferating cell nuclear antigen.

miR-125 mimic group (Fig. 3A). By contrast, a large number of cells migrated into the scratch wound in the miR-125 inhibitor group (Fig. 3B).

To assess the role of miR-125 in apoptosis, RKO cells were transfected with miR-125 mimic or inhibitor or control miR, and apoptosis was determined by caspase- 3 activity, DNA fragmentation and apoptotic morphology. The miR-125 mimic significantly enhanced caspase-3 activity (Fig. 4A) and the inhibitor reduced caspase-3 activity (Fig. 4B). The miR-125 mimic increased DNA fragmentation (Fig. 4C), whereas the inhibitor decreased DNA fragmentation (Fig. 4D). In the miR-125 mimic group, marked morphological features of apoptosis were shown (Fig. 4E). By contrast, there were no notable morphological features of apoptosis in the miR-125 inhibitor group (Fig. 4F). The apoptosis of RKO cells transfected with miR-125 mimic or inhibitor or control miR was measured by flow cytometry. The results showed that the apoptosis of RKO cells transfected with mimics was increased and the population of RKO cells transfected with inhibitor was decreased, compared with that in the control (Fig. 4G).

To determine the effect of miR-125 on the expression of COX-2 and VEGF in RKO cells, the RNA expression of COX-2 was determined using the RT-qPCR method, and the protein levels of COX-2 and VEGF were determined by western blot analysis. The preliminary experiment showed that VEGF was constitutively expressed in the RKO cells (data not shown), therefore, the effect of miR-125 on the expression of VEGF was investigated. The miR-125 mimics caused significant decreases in the expression of COX-2 and VEGF, whereas the miR-125 inhibitors upregulated the expression of COX-2 and VEGF (Fig. 5).
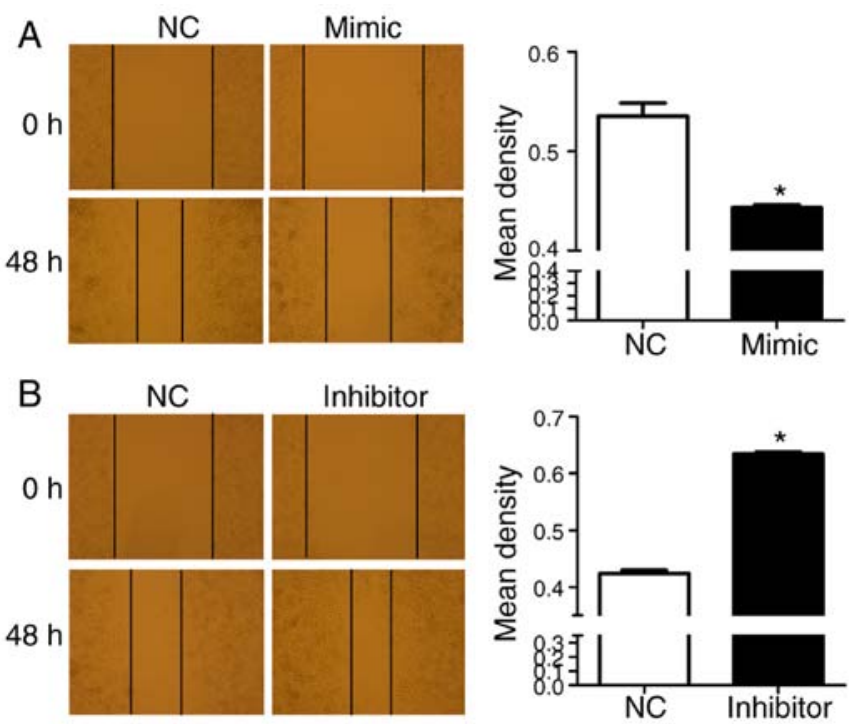

Figure 3. Role of miR-125 in RKO cell migration. RKO cells were transfected with miR-125 mimic, miR-125 inhibitor, or a miR-125 negative control, and cell scratches were observed at 0 and $48 \mathrm{~h}$, respectively. (A) RKO cells transfected with miR-125 mimic or miR-125 negative control. (B) RKO cells transfected with miR-125 inhibitor or miR-125 negative control. ${ }^{*} \mathrm{P}<0.05$ vs. control. miR, microRNA; NC, negative control.

In the development of a primary tumor, uncontrolled growth is a key component. The MAPK pathway is a major pathway associated with uncontrolled growth in CRC, involving proteins which include ERK, p38, JNK and RAS. Several miRNAs have been shown to regulate the proteins involved in this pathway. For example, Let7, miR-143, miR-18a* and miR-145 downregulate 

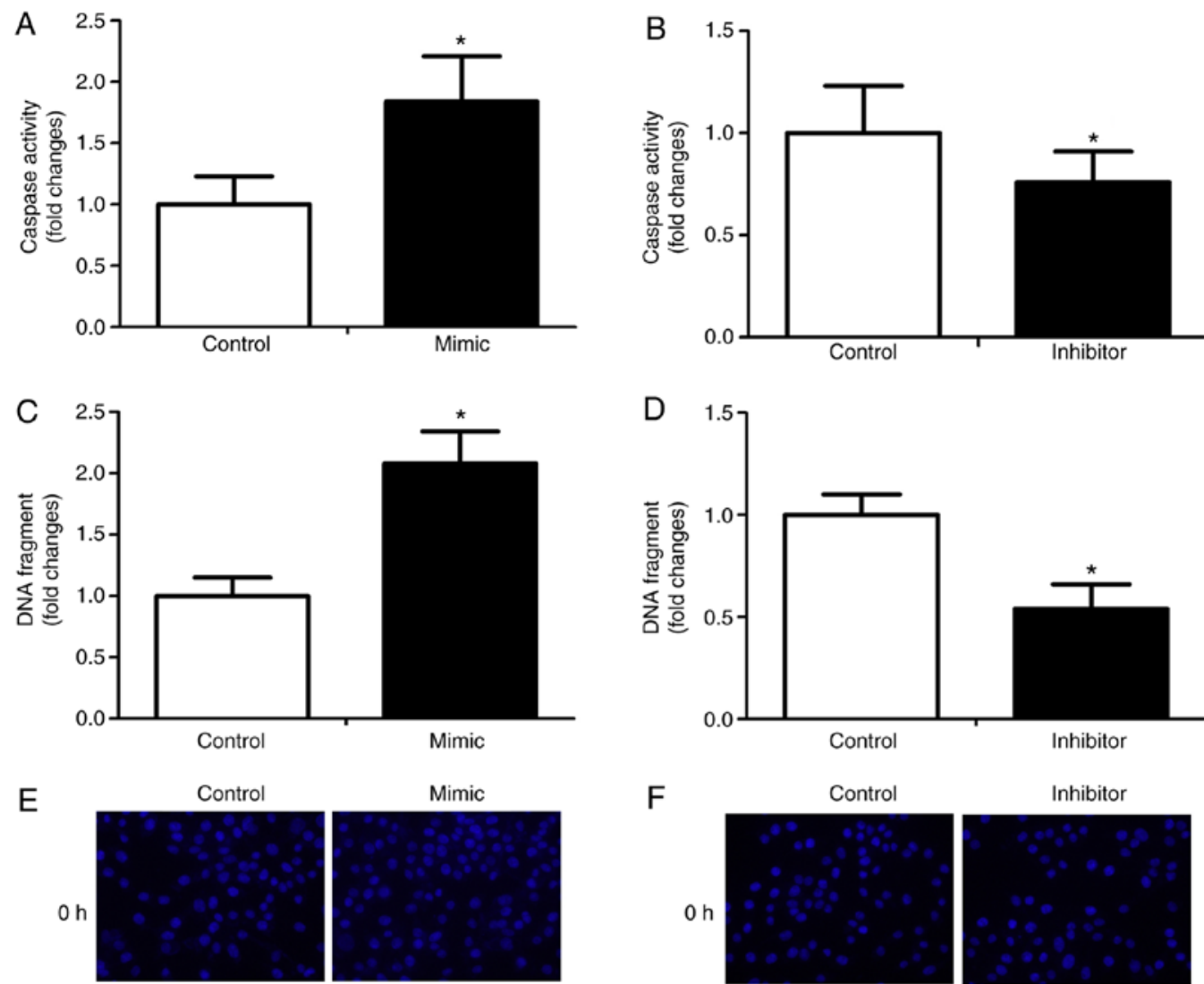

$\mathrm{F}$
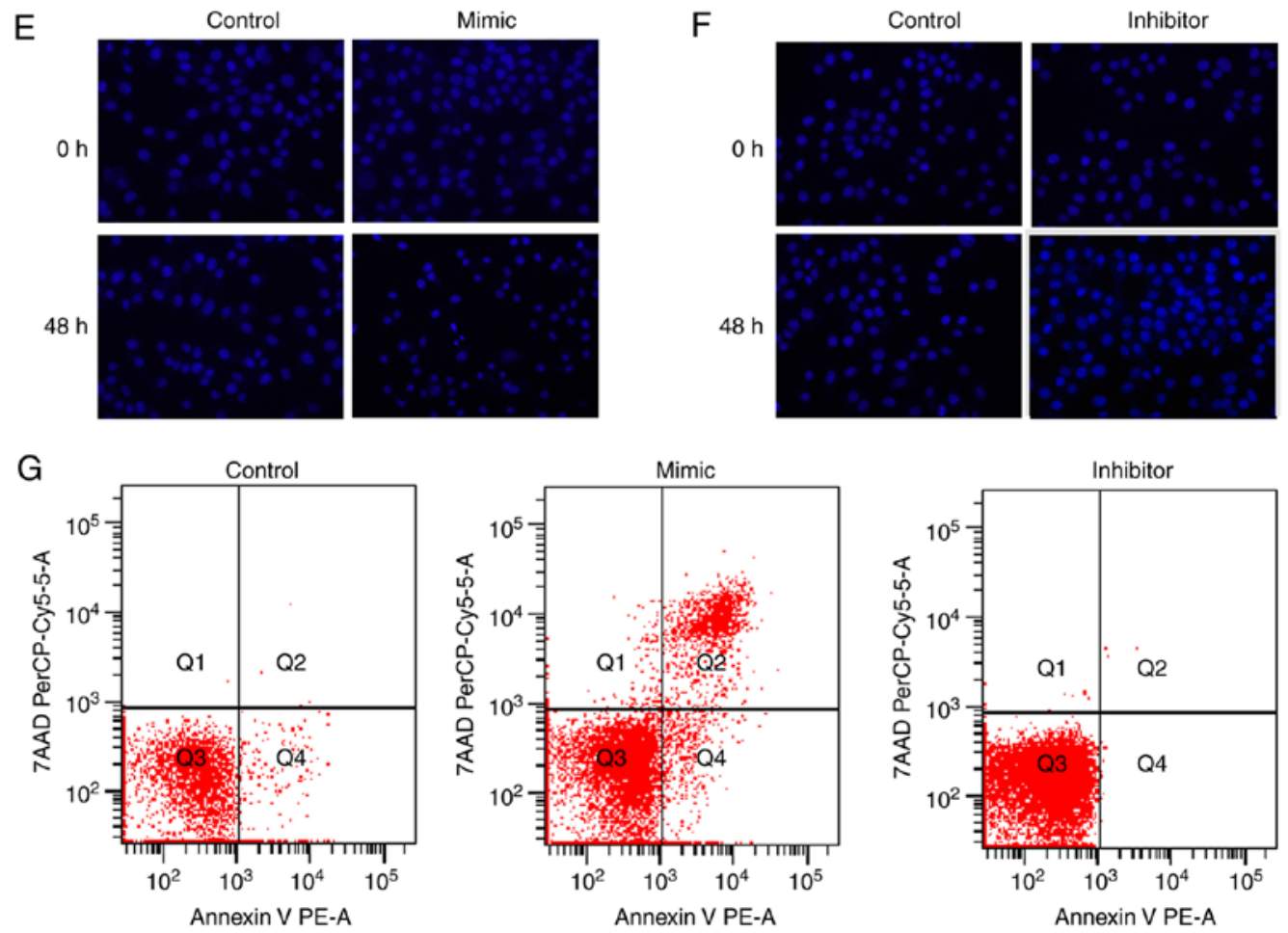

Figure 4. Effect of miR-125 on apoptosis of RKO cells. RKO cells were transfected with miR-125 mimic, inhibitor, or a miR-125 negative control and incubated for $24 \mathrm{~h}$. Apoptosis was determined by an caspase-3 activity, DNA fragmentation and apoptotic morphology. Caspase-3 activity was measured in the (A) mimic and (B) inhibitor groups using a caspase-3 fluorescent assay kit. DNA fragmentation was measured in (C) mimic and (D) inhibitor groups via enzyme-linked immunosorbent assay. Apoptotic morphology was determined in (E) mimic and (F) inhibitor groups using Hoechst (magnification, x200). (G) Apoptosis of RKO cells was measured by flow cytometry. ${ }^{*} \mathrm{P}<0.05$ vs. control. miR, microRNA.

RAS and act as tumor-suppressive miRNAs in CRC (18-21). In the present study, western blot analysis was used to examine the effect of miR-125 on the phosphorylation of ERK, p38 and JNK in RKO cells. The phosphorylation of ERK, p38 and JNK was reduced by the miR-125 mimic, but were increased by the miR-125 inhibitor (Fig. 6).

It was revealed by a dual-luciferase reporter assay that miR-125 functionally interacted with the 3'UTR sequences of VEGF. The alignments between miR-125 and the region within the 3'UTR of VEGF represent putative target sequences which can confer inhibition of translation by miR-125. To assess whether VEGF is a direct target of miR-125, the present study used a reporter vector, which contained a luciferase gene followed by the 3'UTR of VEGF mRNA (wt-Luc-VEGF). The luciferase activity in wt-Luc-VEGF-transfected RKO cells was inhibited by the overexpression of miR-125 by its mimic. To verify this, mutation of the miR-125 putative binding sites on the 3'UTR of VEGF of wt-Luc-VEGF was 

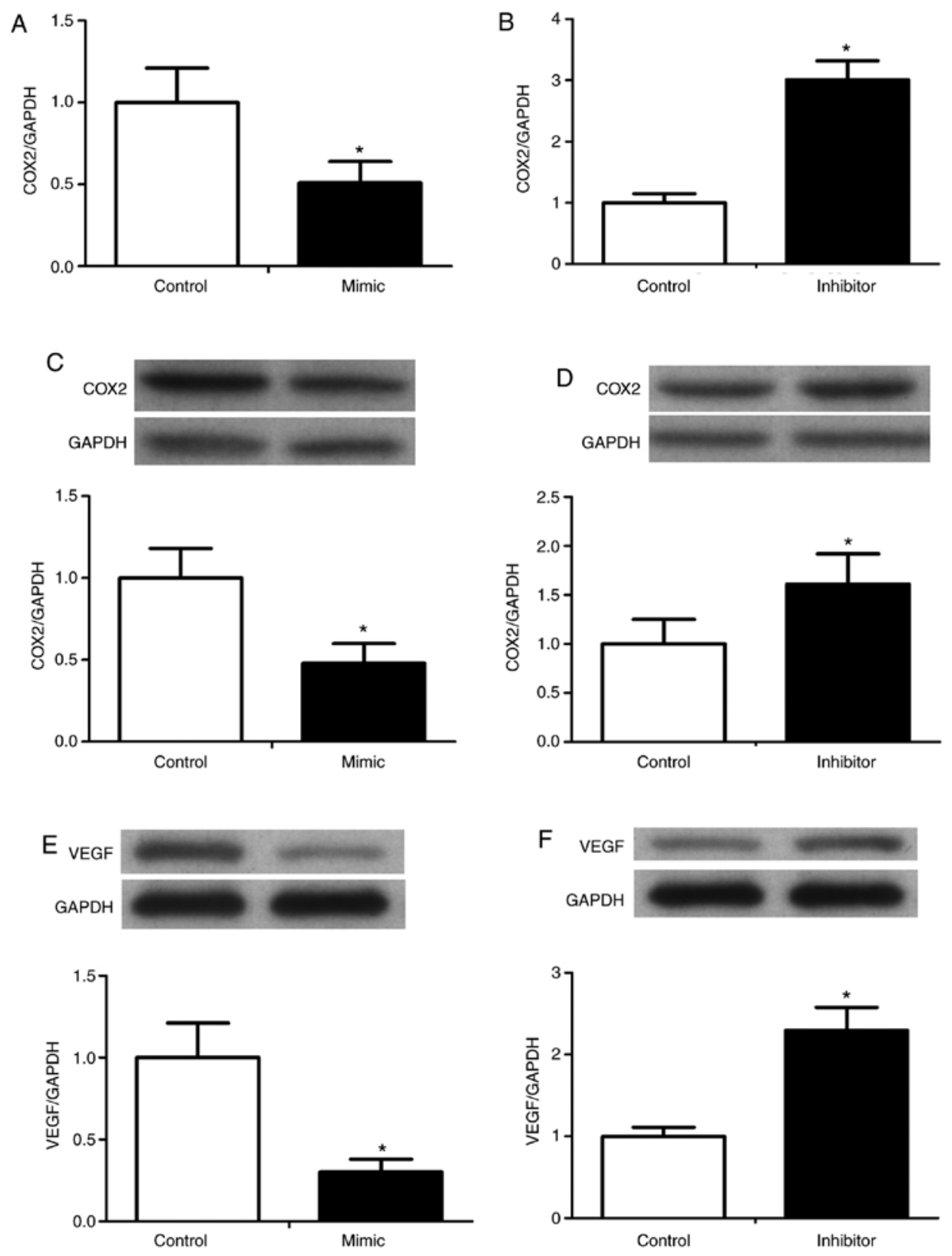

Figure 5. Effect of miR-125 on the expression of COX-2 and VEGF in RKOs. RKOs were transfected with miR-125 mimic, inhibitor, or a miR-125 negative control. (A and B) COX-2 RNA was detected in the (A) mimic and (B) inhibitor groups using RT-qPCR analysis. Protein levels of COX-2 in the (C) mimic and (B) inhibitor groups and GAPDH in the (E) mimic and (F) inhibitor groups were determined by western blot analysis. VEGF RNA was detected using RT-qPCR analysis. Protein levels of VEGF and GAPDH were determined by western blot analysis. "P<0.05 vs. control. miR, microRNA; VEGF, vascular endothelial growth factor; COX-2, cyclooxygenase-2; RT-qPCR, reverse transcription-quantitative polymerase chain reaction.

introduced by substitution (Fig. 7A and B). The mutation abrogated the inhibitory effect of miR-125 on the luciferase activity of the RKO cells (Fig. 7C). By contrast, the inhibition of miR-125 by its inhibitors enhanced the luciferase activity in the wt-Luc-VEGF-transfected RKO cells; the mutation of wt-Luc-VEGF abrogated the enhanced effect of miR-125 on the luciferase activity in RKO cells (Fig. 7D). These data demonstrated that miR-125 directly targeted and inhibited the expression of VEGF in RKO cells.

\section{Discussion}

The major finding of the present study was that induction of the expression of miR-125 promoted the apoptosis of RKO cells.
miR-125 directly targeted VEGF and repressed the expression of VEGF. Therefore, the results of the present study suggested the importance of miR-125 in RKO cells by inducing cell apoptosis.

The associations between miRNAs and malignancies have been investigated in numerous studies, the results of which have shown that miRNA deregulation is involved in all types of cancer. In different types of cancer, different members of the miR-125 family have been reported to have conflicting properties; through acting as either tumor suppressors or oncogenes, they may contribute to the initiation and progression of cancer (22-25).

The tumor-suppressor functions of miR-125 have been shown in several types of cancer, including ovarian 
A
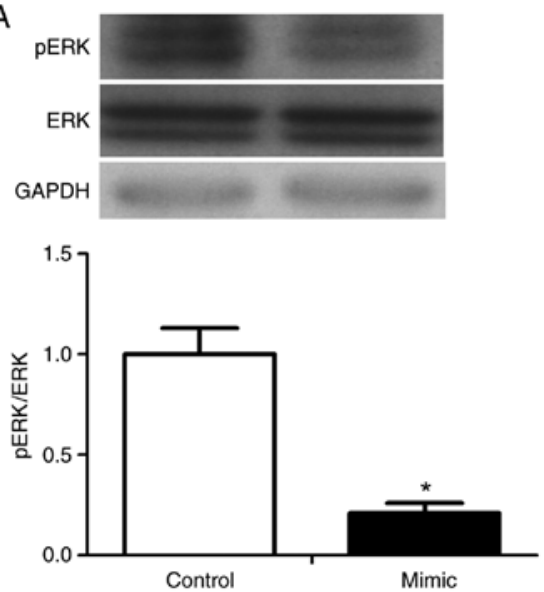

C
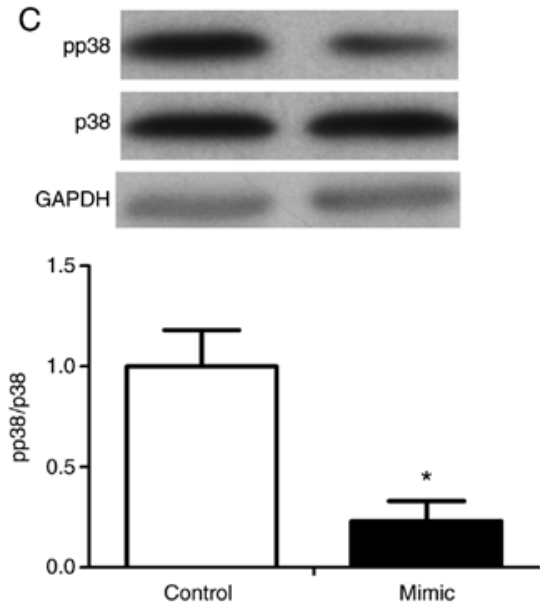

E
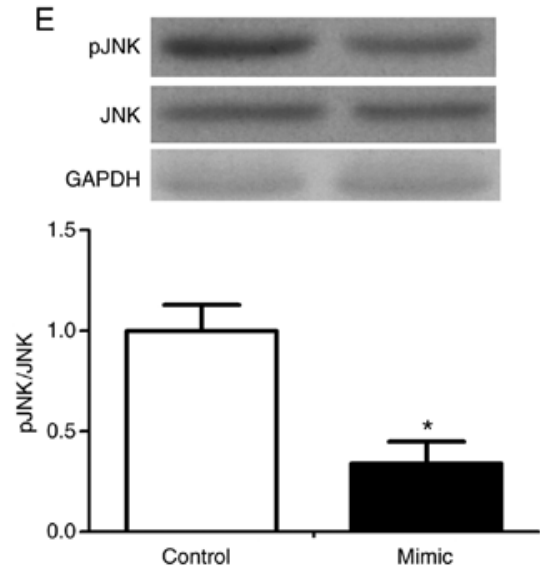

B
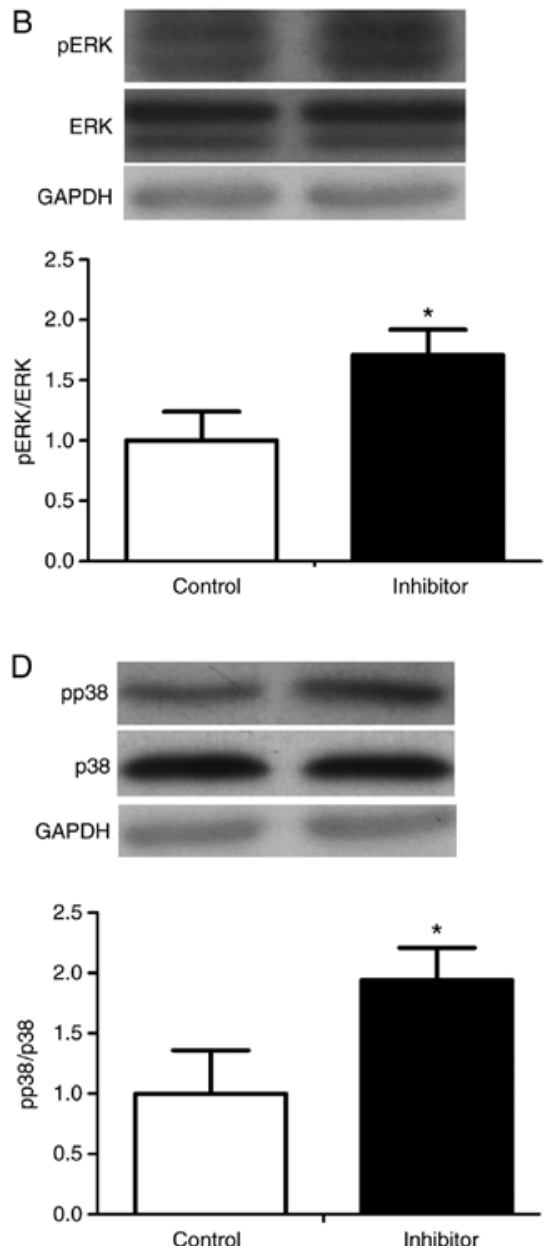

$\mathrm{F}$
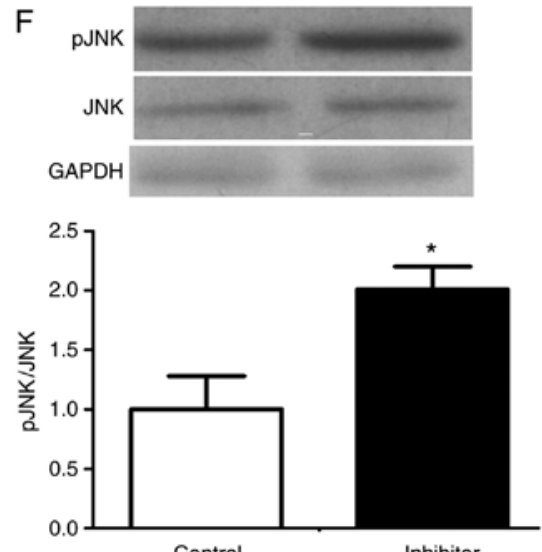

Control

Inhibitor

Figure 6. Effect of miR-125 on the mitogen-activated protein kinase signaling pathway in RKO cells. RKOs were transfected with miR-125 mimic, inhibitor, or a miR-125 negative control. Expression and phosphorylation of ERK in the (A) mimic and (B) inhibitor groups, p38 in the (C) mimic and (D) inhibitor groups, and JNK in the (E) mimic and (F) inhibitor groups were determined by western blot analysis. " $\mathrm{P}<0.05$ vs. control. miR, microRNA; ERK, extracellular signal-regulated kinase; JNK, c-Jun N-terminal kinase; pERK, phosphorylated ERK; pp38, phosphorylated p38; pJNK, phosphorylated JNK.

cancer $(23,26)$, bladder cancer $(27)$, osteosarcoma (28), breast cancer (29-31), hepatocellular carcinoma $(16,32,33)$, melanoma (34) and cutaneous squamous cell carcinoma (35). Chen and Hu (36) used PCR to investigate the level and the methylation status of the miR-125 family in CRC tissues and adjacent non-tumor tissues, and found that miR-125a and miR-125b were significantly downregulated in CRC tissues, and the methylation frequencies of miR-125a and miR-125b were higher in CRC tissues. These results suggested the hypermethylation of miR-125 as a potential biomarker for clinical outcome. In the present study, it was shown that miR-125 also contributed to the apoptosis of CRC cells. In response to CRC, miR-125 inhibitors prevented apoptosis, whereas miR-125 mimics enhanced apoptosis. As VEGF stimulates angiogenesis, proliferation and migration, it has been implicated in tumor generation (37). In the present study, the induction of miR-125 inhibited the expression of VEGF, whereas the inhibition of miR-125 enhanced the expression 
A
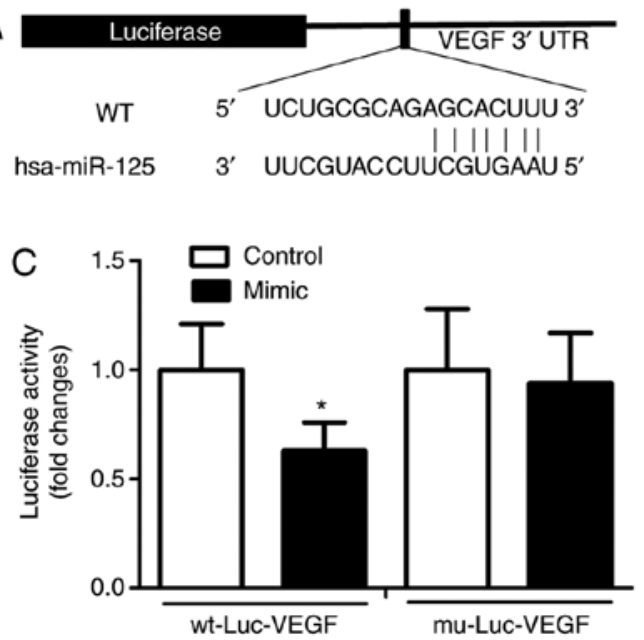

B

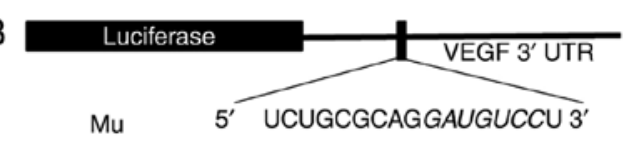

hsa-miR-125 3' UUCGUACCUUCGUGAAU 5'

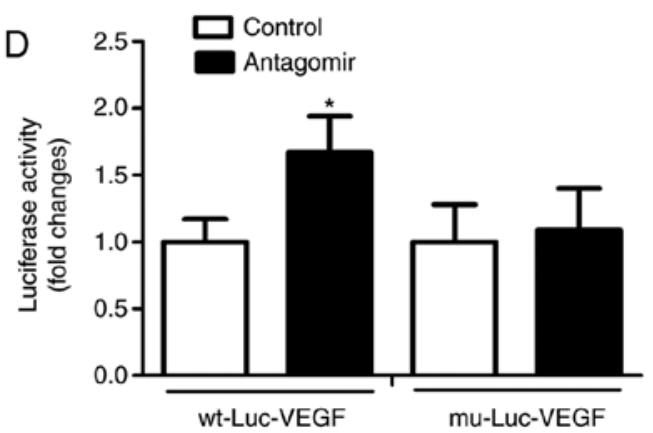

Figure 7. miR-125 targets and inhibits VEGF. (A) A segment of VEGF 3'UTR was inserted downstream of the luciferase-coding sequence. The complementarity at the 5' end of miR-125 was shown by the sequence alignment of miR-125 and the 3'UTR of VEGF. (B) Sequence alignment of miR-125 and mutated 3'UTR of VEGF showed no complementarity at the 5' end of miR-125. (C) RKO cells were transfected with the plasmid containing the wt-Luc-VEGF or mu-Luc-VEGF, and miR-125 mimic or a miR-125 negative control. (D) RKO cells were transfected with the plasmid containing wt-Luc-VEGF or mu-Luc-VEGF, and miR-125 inhibitor or a miR-125 negative control. "P<0.05 vs. control. miR, microRNA; VEGF, vascular endothelial growth factor; UTR, untranslated region; wt-Luc-VEGF, wild-type 3'UTR of VEGF; mu-Luc-VEGF, mutated 3'UTR of VEGF.

of VEGF. This confirmed that miR-125 directly targeted and inhibited the expression of VEGF in RKO cells. Substantial data support the use of targeted agents directed against the molecular signaling pathways involved in angiogenesis, particularly the VEGF pathway (38). MAPK is a type of VEGF signaling pathway and MAPK has three signaling pathways: ERK, p38 and JNK. It is known that ERK, JNK and p38 transduce the signal through phosphorylation. Martínez-Acuña et al determined the expression of miR-125a-5p from nine cervical cell lines using RT-PCR analysis and found that miR-125a-5p was involved in the migration of cervical cancer tumor cells via MAPK1 as a functional target (12). The present study demonstrated that miR-125 mimics decreased the phosphorylation of ERK, p38 and JNK, whereas miR-125 inhibitors increased this phosphorylation. It is known that the majority of cases of cancer-associated mortality are due to infection and inflammation. COX-2 is a type of inflammatory maker. In previous years, several studies have found that the miR-125 family is involved in cancer inflammation by regulation of the RNA-binding protein HuR (39), and COX-2 is an important target in various tumors $(40,41)$. In the present study, it was shown that miR-125 mimics significantly decreased the expression of COX-2, whereas miR-125 inhibitors upregulated the expression of COX-2.

Although the present study revealed interesting results, there were a number of limitations. Firstly, the investigation only used luciferase assays to investigate the targeting association between miR-125 and VEGF, with no western blot or RT-PCR assays performed to confirm the association. Secondly, the levels of miR-125 were not examined in CRC tissues in the present study, therefore, future investigations require the levels of miRNA-125 to be detected in CRC tissues. Thirdly, the levels of non-phosphorylated protein are expected to decrease accordingly when phosphorylation occurs. However, there was no change in the level of non-phosphorylated protein in the present study, the reason for which remains to be elucidated, as does the reason for miR-125 affecting the phosphorylation of MAPK. Additionally, only in vitro experiments involving only one cell line were performed, which is insufficient for a solid conclusion. In subsequent investigations, the use of in vivo experiments and animal models id required to confirm the conclusion.

In conclusion, the present study confirmed that miR-125 was important in RKO cells and VEGF was confirmed as a direct target of miR-125. The promotion effect of miR-125 on apoptosis was mediated through reducing the expression of VEGF. Therefore, miR-125 may be a novel therapeutic target for CRC.

\section{Acknowledgements}

Not applicable.

\section{Funding}

The present study was supported by National Natural Science Foundation of China (grant nos. 81570419 and 81270372).

\section{Availability of data and materials}

All data generated or analyzed during this study are included in this published article.

\section{Author's contributions}

QBW, JC, JWZ, XY, HYY and YRL made substantial contributions to conception and design, acquisition of data, analysis and interpretation of data. HQZ was involved in drafting the manuscript or revising it critically for important intellectual content and gave final approval of the version to be published. HQZ also agreed to be accountable for all aspects of the work in ensuring that questions related to the accuracy or integrity of any part of the work are appropriately investigated and resolved. 


\section{Ethics approval and consent to participate}

Not applicable.

\section{Consent for publication}

Not applicable.

\section{Competing interests}

The authors declare that they have no competing interests.

\section{References}

1. Haggar FA and Boushey RP: Colorectal cancer epidemiology: Incidence, mortality, survival, and risk factors. Clin Colon Rectal Surg 22: 191-197, 2009.

2. Siegel R, Desantis C and Jemal A: Colorectal cancer statistics, 2014. CA Cancer J. Clin 64: 104-117, 2014.

3. Dean M: Cancer as a complex developmental disorder-nineteenth Cornelius P. Rhoads memorial award lecture. Cancer Res 58 5633-5636, 1998.

4. Bartel DP: MicroRNAs: Genomics, biogenesis, mechanism, and function. Cell 116: 281-297, 2004.

5. Meister $\mathrm{G}$ and Tuschl T: Mechanisms of gene silencing by double-stranded RNA. Nature 431: 343-349, 2004.

6. Kumarswamy $\mathrm{R}$ and Thum T: Non-coding RNAs in cardiac remodeling and heart failure. Circ Res 113: 676-689, 2013.

7. Quiat D and Olson EN: MicroRNAs in cardiovascular disease: From pathogenesis to prevention and treatment. J Clin Invest 123 11-18,2013.

8. Bartel DP: MicroRNAs: Target recognition and regulatory functions. Cell 136: 215-233, 2009.

9. Huntzinger E and Izaurralde E: Gene silencing by microRNAs: Contributions of translational repression and mRNA decay. Nat Rev Genet 12: 99-110, 2011.

10. Winter J, Jung S, Keller S, Gregory RI and Diederichs S: Many roads to maturity: MicroRNA biogenesis pathways and their regulation. Nat Cell Biol 11: 228-234, 2009.

11. Pillai RS, Bhattacharyya SN and Filipowicz W: Repression of protein synthesis by miRNAs: How many mechanisms? Trends Cell Biol 17: 118-126, 2007.

12. Martínez-Acuña N, González-Torres A, Tapia-Vieyra JV and Alvarez-Salas LM: MARK1 is a novel target for miR-125a-5p: Implications for cell migration in cervical tumor cells. Microrna, 2017.

13. Yin H, Sun Y, Wang X, Park J, Zhang Y, Li M, Yin J, Liu Q and Wei M: Progress on the relationship between miR-125 family and tumorigenesis. Exp Cell Res 339: 252-260, 2015.

14. Aldebasi YH, Rahmani AH, Khan AA and Aly SM: The effect of vascular endothelial growth factor in the progression of bladder cancer and diabetic retinopathy. Int J Clin Exp Med 6: 239-251, 2013.

15. Stiegelbauer V, Perakis S, Deutsch A, Ling H, Gerger A and Pichler M: MicroRNAs as novel predictive biomarkers and therapeutic targets in colorectal cancer. World J Gastroenterol 20 : $11727-11735,2014$

16. Bi Q, Tang S, Xia L, Du R, Fan R, Gao L, Jin J, Liang S, Chen Z, $\mathrm{Xu} \mathrm{G}$, et al: Ectopic expression of miR-125a inhibits the proliferation and metastasis of hepatocellular carcinoma by targeting MMP11 and VEGF. PLos One 7: e40169, 2012.

17. Livak KJ and Schmittgen TD: Analysis of relative gene expression data using real-time quantitative PCR and the 2-(delta delta C(T)) method. Methods 25: 402-408, 2001.

18. Sun X, Zhang S and Ma X: Prognostic value of MicroRNA-125 in various human malignant neoplasms: A meta-analysis. Clin Lab 61: 1667-1674, 2015.

19. Johnson SM, Grosshans H, Shingara J, Byrom M, Jarvis R, Cheng A, Labourier E, Reinert KL, Brown D and Slack FJ: RAS is regulated by the let-7 microRNA family. Cell 120: 635-647, 2005.

20. Tsang WP and Kwok TT: The miR-18a* microRNA functions as a potential tumor suppressor by targeting on K-Ras. Carcinogenesis 30: 953-959, 2009.
21. Chen X, Guo X, Zhang H, Xiang Y, Chen J, Yin Y, Cai X, Wang K, Wang G, Ba Y, et al: Role of miR-143 targeting KRAS in colorectal tumorigenesis. Oncogene 28: 1385-1392, 2009.

22. Yin Y, Yan ZP, Lu NN, Xu Q, He J, Qian X, Yu J, Guan X, Jiang BH and Liu L Z: Downregulation of miR-145 associated with cancer progression and VEGF transcriptional activation by targeting N-RAS and IRS1. Biochim Biophys Acta 1829: 239-247, 2013.

23. Bousquet M, Harris MH, Zhou B and Lodish HF: MicroRNA miR-125b causes leukemia. Proc Natl Acad Sci USA 107: 21558-21563, 2010

24. Cowden Dahl KD, Dahl R, Kruichak JN and Hudson LG: The epidermal growth factor receptor responsive miR-125a represses mesenchymal morphology in ovarian cancer cells. Neoplasia 11: 1208-1215, 2009.

25. Jiang L, Huang Q, Zhang S, Zhang Q, Chang J, Qiu X and Wang E: Hsa-miR- 125a-3p and hsa-miR-125a-5p are downregulated in non-small cell lung cancer and have inverse effects on invasion and migration of lung cancer cells. BMC Cancer 10: 318, 2010.

26. Jiang F, Liu T, He Y, Yan Q, Chen X, Wang $H$ and Wan $X$ MiR-125b promotes proliferation and migration of type II endometrial carcinoma cells through targeting TP53INP1 tumor suppressor in vitro and in vivo. BMC Cancer 11: 425, 2011.

27. Guan Y, Yao H, Zheng Z, Qiu G and Sun K: MiR-125b targets BCL3 and suppresses ovarian cancer proliferation. Int J Cancer 128: 2274-2283, 2010.

28. Huang L, Luo J, Cai Q, Pan Q, Zeng H, Guo Z, Dong W, Huang J and Lin T: MicroRNA-125b suppresses the development of bladder cancer by targeting E2F3. Int J Cancer 128: 1758-1769, 2011

29. Liu LH, Li H, Li JP, Zhong H, Zhang HC, Chen J and Xiao T: MiR-125b suppresses the proliferation and migration of osteosarcoma cells through down-regulation of STAT3. Biochem Biophys Res Commun 416: 31-38, 2011.

30. Li W, Duan R, Kooy F, Sherman SL, Zhou W and Jin P: Germline mutation of microRNA-125a is associated with breast cancer. J Med Genet 46: 358-360, 2009.

31. Scott GK, Goga A, Bhaumik D, Berger CE, Sullivan CS and Benz CC: Coordinate suppression of ERBB2 and ERBB3 by enforced expression of micro-RNA miR-125a or miR-125b. J Biol Chem 282: 1479-1486, 2007.

32. Mattie MD, Benz CC, Bowers J, Sensinger K, Wong L, Scott GK, Fedele V, Ginzinger D, Getts R and Haqq C: Optimized high-throughput microRNA expression profiling provides novel biomarker assessment of clinical prostate and breast cancer biopsies. Mol Cancer 5: 24, 2006.

33. Liang L, Wong CM, Ying Q, Fan DN, Huang S, Ding J, Yao J, Yan M, Li J, Yao M, et al: MicroRNA-125b suppressesed human liver cancer cell proliferation and metastasis by directly targeting oncogene LIN28B2. Hepatology 52: 1731-1740, 2010.

34. Jia HY, Wang YX, Yan WT, Li HY, Tian YZ, Wang SM and Zhao HL: MicroRNA-125b functions as a tumor suppressor in hepatocellular carcinoma cells. Int J Mol Sci 13: 8762-8774, 2012.

35. Kappelmann M, Kuphal S, Meister G, Vardimon L and Bosserhoff AK: MicroRNA miR-125b controls melanoma progression by direct regulation of c-Jun protein expression. Oncogene 32: 2984-2991, 2013.

36. Chen $\mathrm{H}$ and $\mathrm{Hu} \mathrm{Z}$ : Hypermethylation-associated silencing of miR-125a and miR-125b: A potential marker in colorectal cancer. Dis Markers 2015: 345080, 2015.

37. Nagy, JA, Dvorak AM and Dvorak HF: VEGF and the induction of pathological angiogenesis. Annu Rev Pathol 2: 251-275, 2007.

38. Aragon-Ching JB and Dahut WL: Anti-angiogenesis approach to genitourinary cancer treatment. Update Cancer Ther 3: 182-188, 2009.

39. Dormoy-Raclet V, Ménard I, Clair E, Kurban G, Mazroui R, Di Marco S, von Roretz C, Pause A and Gallouzi IE: The RNA-binding protein HuR promotescell migration and cell invasion by stabilizing the beta-actin mRNA in a U-rich-element-dependent manner. Mol Cell Biol 27: 5365-5380, 2007.

40. Denkert C, Weichert W, Pest S, Koch I, Licht D, Köbel M, Reles A, Sehouli J, Dietel M and Hauptmann S: Overexpression of the embryonic-le-thal abnormal vision-like protein HuR in ovarian carcinoma is a prognostic factor and is associated with increased cyclooxygenase-2expression. Cancer Res 64: 189-195, 2004.

41. Mrena J, Wiksten JP, Thiel A, Kokkola A, Pohjola L, Lundin J, Nordling S, Ristimäki A and Haglund C: Cyclooxygenase-2 is an independent prognostic factor in gastric cancer and its expression is regulated by the messenger RNA stability factor HuR. Clin Cancer Res 11: 7362-7368, 2005. 